\title{
Dihydroartemisinin-induced inhibition of proliferation in BEL-7402 cells: An analysis of the mitochondrial proteome
}

\author{
JIN-JIAN LU ${ }^{1,2^{*}}$, ZHEN YANG $^{1 *}$, DE-ZHAO LU ${ }^{1}$, XING-DE WO $^{1}$, JIA-JIE SHI ${ }^{1}$, \\ TAO-QI LIN ${ }^{1}$, MI-MI WANG ${ }^{1}$, YI LI ${ }^{1}$ and LI-HUA TANG ${ }^{1}$ \\ ${ }^{1}$ College of Life Sciences, Zhejiang Chinese Medical University, Hangzhou, Zhejiang 310053; \\ ${ }^{2}$ State Key Laboratory of Quality Research in Chinese Medicine, Institute of Chinese Medical Sciences, \\ University of Macau, Macao SAR, P.R. China
}

Received January 25, 2012; Accepted May 2, 2012

DOI: $10.3892 / \mathrm{mmr} .2012 .906$

\begin{abstract}
Artemisinin, the active ingredient of the Chinese medicinal herb Artemisia annua L., and its derivatives (ARTs) are currently widely used as anti-malarial drugs around the world. In this study, we found that dihydroartemisinin (DHA), one of the main active metabolites of ARTs, inhibited the proliferation of human hepatocarcinoma BEL-7402 cells in a concentration-dependent manner. To interpret the mechanisms involved, an analysis of the mitochondrial proteome was performed employing two-dimensional gel electrophoresis and matrix-assisted laser desorption/ionization time-of-flight mass spectrometry. Seven mitochondrial proteins including fumarate hydratase, $60 \mathrm{kDa}$ heat shock protein, enoyl-CoA hydratase, 3-hydroxyacyl-CoA dehydrogenase, two subunits of ATP synthase and NADPH:adrenodoxin oxidoreductase were identified to be differentially expressed between the control and DHA-treated groups. Our results indicate that the imbalance of energy metabolism induced by DHA may contribute, at least in part, to its anti-cancer potential in BEL-7402 cells.
\end{abstract}

Correspondence to: Dr Jin-Jian Lu, College of Life Sciences, Zhejiang Chinese Medical University, 548 Binwen Road, Binjiang District, Hangzhou, Zhejiang 310053, P.R. China

E-mail: jinjian.lu@163.com

*Contributed equally

Abbreviations: ARTs, artemisinin and its derivatives; 2-DE, two-dimensional gel electrophoresis; DHA, dihydroartemisinin; ECAH, enoyl-CoA hydratase; FH, fumarate hydratase; GRP78, glucose-regulated protein 78; HCAD, 3-hydroxyacyl-CoA dehydrogenase; IEF, isoelectric focusing; HIF, hypoxia-inducible transcription factor; HSP60, $60 \mathrm{kDa}$ heat shock protein; MALDI-TOF MS, matrix-assisted laser desorption/ionization time-of-flight mass spectrometry; NAO, NADPH:adrenodoxin oxidoreductase; SRB, sulforhodamine B

Key words: hepatocarcinoma, dihydroartemisinin, mitochondria, proteomics, energy metabolism

\section{Introduction}

Traditional Chinese medicine is one of the important resources for the development of modern drugs. Artemisinin (Fig. 1A), the active component of the Chinese medicinal herb Artemisia annua L., and its derivatives (ARTs) are used extensively as anti-malarial drugs worldwide (1). ARTs have also been studied as candidates for cancer therapy, as they effectively inhibit proliferation in various cancer cells (2-8). It appears that ironmediated cleavages of the endoperoxide bridge play a vital role in achieving their anti-cancer effects $(9,10)$. Pre-treatment with an iron chelator reverses the proliferative inhibition of ARTs, while pre-loading cancer cells with iron or holotransferrin enhances the cytotoxicity $(2,11,12)$. ARTs lead to cell cycle arrest $(3,6,8,13)$, apoptosis $(2,8,14-16)$ or oncosis-like cell death (17) in various cell types. Recently, proteomics were used to further elucidate the molecular mechanisms of ARTs, and the investigators found that the endoplastic reticulum stress induced by dihydroartemisinin (DHA, Fig. 1B), one of the main active metabolites of ARTs, partially contributed to its anti-cancer activity (13). However, as the authors proposed, a sub-cellular proteomics study is required to avoid the biases of whole cellular proteomics, such as the neglect of low-expressing proteins (13).

Mitochondria generate the majority of the chemical energy in eukaryotic cells and are involved in a series of physiological processes, such as signaling transduction and cell cycle processes, as well as the control of cell death. Previous studies indicated that mitochondria are important in the pharmacological activities of ARTs $(3,18)$. The aim of this study was to detect the differentially expressed mitochondrial proteins using proteomics to recognize the possible mechanisms involved.

In this study, we found that DHA effectively triggered cell proliferative inhibition in human hepatocellular carcinoma BEL-7402 cells. By profiling the altered protein expression pattern following DHA treatment, seven differentially expressed mitochondrial proteins were identified between the control and DHA-treated groups.

\section{Materials and methods}

Materials. DHA was kindly provided by Professor Ying Li, Shanghai Institute of Materia Medica, Chinese Academy of 
Sciences (Shanghai, China). Sulforhodamine B (SRB) was obtained from Sigma Chemical Co. (St. Louis, MO, USA).

Cell line and culture. The human hepatocellular carcinoma BEL-7402 cell line was provided by Professor Jian Ding, Shanghai Institute of Materia Medica, Chinese Academy of Sciences (Shanghai, China) and maintained in RPMI-1640 (Sigma) supplemented with $10 \%$ heat-inactivated fetal bovine serum (Sijiqing, Hangzhou, China). Cells were incubated in a humidified atmosphere of $95 \%$ air plus $5 \% \mathrm{CO}_{2}$ at $37^{\circ} \mathrm{C}$.

SRB assay. The effect of DHA on cell proliferation was determined by a SRB assay as previously described (13). Briefly, BEL-7402 cells were seeded into 96-well plates and treated with the indicated concentrations of DHA for $48 \mathrm{~h}$. The medium was discarded immediately following the drug treatment, and the cells were fixed with TCA. After the plate was washed and dried, SRB was added. The plate was washed, dried and the SRB retained in the cells was dissolved in $150 \mu \mathrm{l}$ of $10 \mathrm{mM}$ Tris-HCl. The OD values at $515 \mathrm{~nm}$ were then measured using a multi-well plate reader (SpectraMax Microplate Reader Series 2, Molecular Devices, Sunnyvale, CA, USA).

Observation of morphologic changes. BEL-7402 cells were seeded into 96-well plates and treated with the indicated concentration of DHA for $48 \mathrm{~h}$. The cell morphology was observed using a microscope (NIB-100, NOVEL, China).

Protein sample preparation. BEL-7402 cells were treated with $50 \mu \mathrm{M}$ DHA for $24 \mathrm{~h}$ and the mitochondria were extracted according to the manufacturer's instructions (C3601, Beyotime, China). The mitochondria component was lysed in $200 \mu 1$ lysis solution (30 mM Tris-HCl, $8 \mathrm{M}$ urea, $4 \%$ CHAPS, $\mathrm{pH} 8.5$ ) for $30 \mathrm{~min}$ at $4^{\circ} \mathrm{C}$ and centrifuged at $12,000 \mathrm{~g}$ for $20 \mathrm{~min}$ at $4^{\circ} \mathrm{C}$. Finally, the protein concentrations in the suspended solutions were determined by the Amersham 2D QuantKit (GE Healthcare).

Two-dimensional gel electrophoresis (2-DE). A total of $300 \mu \mathrm{g}$ protein was resolved in $450 \mu \mathrm{l}$ rehydration solution (8 M Urea, 2\% CHAPS, 20 mM DTT, 0.5\% IPG buffer, trace amount of bromophenol blue), loaded onto the $24 \mathrm{~cm}$ immobiline dry strips (pH 3-10, NL, GE Healthcare), and incubated for $12 \mathrm{~h}$. Isoelectric focusing (IEF) was performed on the $\mathrm{Ettan}^{\mathrm{TM}}$ IPGPhor (Amersham) and focused for $12 \mathrm{~h}$ at $30 \mathrm{~V}$, $1 \mathrm{~h}$ at $500 \mathrm{~V}, 1 \mathrm{~h}$ at $1,000 \mathrm{~V}$, and $8,000 \mathrm{~V}$ for $64,000 \mathrm{Vhr}$. The strips were immediately equilibrated twice for $15 \mathrm{~min}$ each using a solution containing $50 \mathrm{mM}$ Tris-HCL, $\mathrm{pH} 8.8$, 6 M Urea, 30\% glycerol, $2 \%$ SDS and $0.002 \%$ bromophenol blue. Subsequently, $1 \%$ DTT and $2.5 \%$ iodoacetamide were added into the first and second equilibration steps, respectively. Following equilibration, the strips were loaded onto a $12.5 \%$ homogeneous SDS polyacrylamide gel and resolved in the second dimension in the Ettan ${ }^{\mathrm{TM}}$ DALT Six (Amersham). The proteins on the gels were visualized by the silver stain method. All 2-DE images were scanned with the Typhoon 9400 (Amersham). Image Master 2D 5.0 software was used for matching and analysis of the protein spots. The spots with an average increase or decrease of 1.8 -fold compared with that of the control group were used.
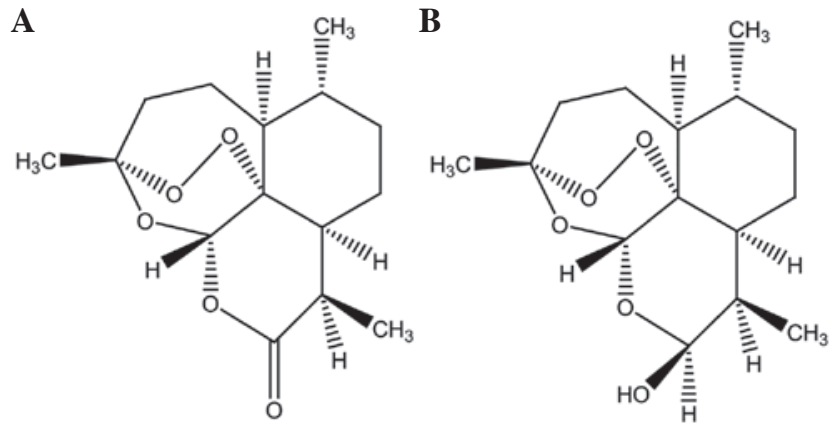

Figure 1. The chemical structures of (A) artemisinin and (B) dihydroartemisinin (DHA)

In-gel digestion and matrix-assisted laser desorption/ionization time-of-flight mass spectrometry (MALDI-TOF MS). For identification, each protein spot of interest was excised from the gels, and destained by washing with $50 \mu \mathrm{l}$ destaining solution $\left[50 \mathrm{mM} \mathrm{Na}_{2} \mathrm{~S}_{2} \mathrm{O}_{3}, 15 \mathrm{mM} \mathrm{K}_{3} \mathrm{Fe}(\mathrm{CN})_{6}\right]$ for $10 \mathrm{~min}$. The spots were then washed with $200 \mu 1 \mathrm{NH}_{4} \mathrm{HCO}_{3}(200 \mathrm{mM})$ for $10 \mathrm{~min}$. The gel spots were dehydrated by acetonitrile for $8 \mathrm{~min}$. Subsequently, they were enzymolysed using $12.5 \mathrm{ng} / \mu \mathrm{l}$ trypsin at $37^{\circ} \mathrm{C}$ overnight. The peptides were extracted twice using extract liquor $\left(\mathrm{H}_{2} \mathrm{O}\right.$ :TFA:acetonitrile $\left.=45: 5: 50\right)$ and freeze dried. In total, $1.2 \mu 1$ eluent containing $0.1 \%$ TFA was added into the peptides and $0.3 \mu \mathrm{l}$ sample solution was analyzed by MALDI-TOF MS (ABI 4800 Plus). The protein identification was performed by searching the NCBI database using the MASCOT search engine.

\section{Results}

Effects of DHA on the proliferation of BEL-7402 cells. Human hepatocarcinoma BEL-7402 cells were treated with various concentrations of DHA for $48 \mathrm{~h}$ and the cell proliferation was evaluated by SRB assay. DHA was found to inhibit cell proliferation in a concentration-dependent manner (Fig. 2A). Morphological changes were also observed following DHA treatment. DHA decreased the percentage of adherent cells in a concentration-dependent manner in BEL-7402 cells (Fig. 2B).

Mitochondria proteomics analysis. Since mitochondria may be involved in the pharmacological activity of ARTs as previously reported $(3,18)$, the mitochondrial proteomics were used to explore the molecular mechanisms of action of DHA in BEL-7402 cells. 2-DE plus MALDI-TOF MS analysis were conducted to compare the mitochondrial protein expression profiles between control and DHA-treated cells. BEL-7402 cells were treated with $50 \mu \mathrm{M}$ DHA for $24 \mathrm{~h}$ to trigger a cell response and the mitochondrial proteins were extracted and separated with 2-D gel as described in Materials and methods (Fig. 3A). The magnified differentially expressed protein spots are shown in Fig. 3B. According to the results analyzed by Image Master 2D 5.0 software, several differentially expressed proteins ( $>1.8$-fold) were further identified by MALDI-TOF MS analysis following DHA treatment. Three mitochondrial proteins [fumarate hydratase $(\mathrm{FH}), 60 \mathrm{kDa}$ heat shock protein (HSP60), enoyl-CoA hydratase (ECAH)] were upregulated and four [3-hydroxyacyl-CoA dehydrogenase (HCAD), two 

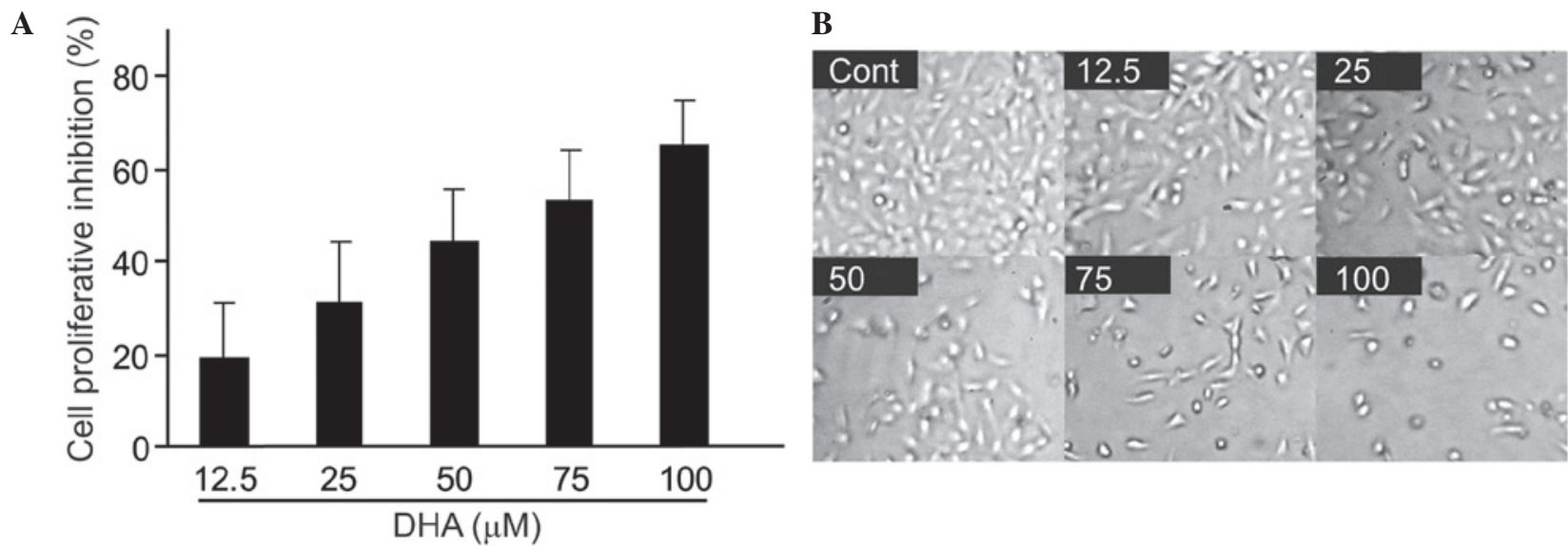

Figure 2. Effects of DHA on the proliferation of BEL-7402 cells. (A) BEL-7402 cells were treated with various concentrations of DHA for $48 \mathrm{~h}$ and the cell proliferation was determined by SRB assay. (B) The morphological changes of BEL-7402 cells following DHA treatment. Cells were treated with various concentrations of DHA for $48 \mathrm{~h}$ and images were captured. DHA, dihydroartemisinin; SRB, sulforhodamine B.

A

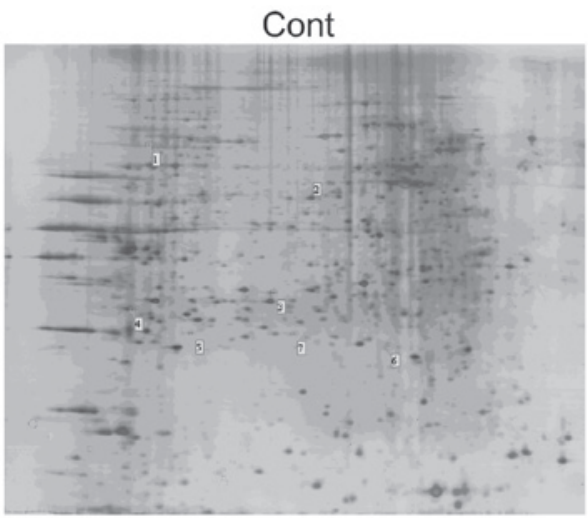

DHA

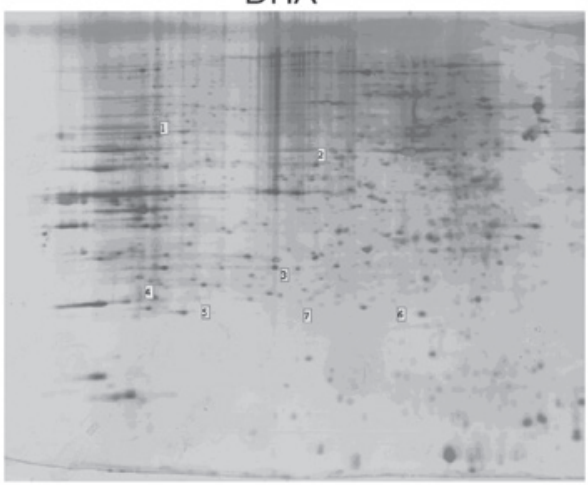

B

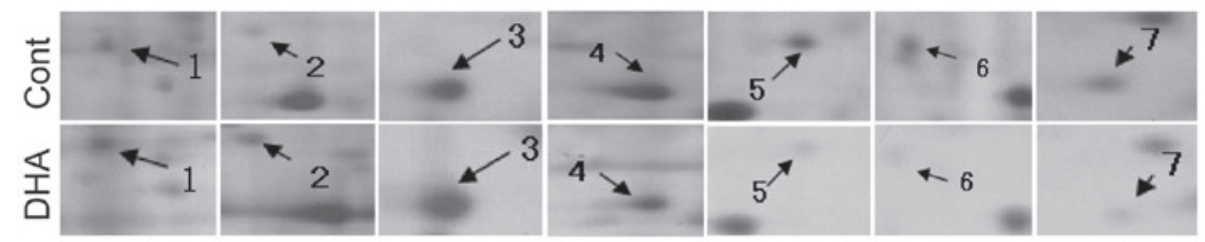

Figure 3. The proteomics analysis. (A) The 2-DE gel image for the protein samples from control and DHA-treated BEL-7402 cells. BEL-7402 cells were treated with $50 \mu \mathrm{M}$ DHA for $24 \mathrm{~h}$ and the mitochondria were extracted and lysed. (B) The magnified differentially expressed protein spots. 2-DE, two-dimensional gel electrophoresis; DHA, dihydroartemisinin.

subunits of ATP synthase and NADPH:adrenodoxin oxidoreductase (NAO)] were downregulated following DHA treatment (Table I). The majority of the proteins were correlated with energy metabolism, indicating the vital role of mitochondria in the anti-cancer activities of DHA. The MALDI-TOF-MS peptide maps of in-gel tryptic digest resulting from HSP60 (spot 2) and ATP synthase subunit d (spot 6) are shown in Fig. 4.

\section{Discussion}

A previous study reported that endoplasmic reticulum stress is important in DHA-induced cell proliferative inhibition in colorectal carcinoma HCT116 cells by using proteomics (13). To the best of our knowledge, this is the first time that mitochondrial proteomics has been performed to search for differentially expressed proteins in human hepatocellular carcinoma BEL-7402 cells following DHA treatment.
In the present study, seven mitochondrial proteins differentially expressed in the control and DHA-treated groups were detected. Among them, three proteins were upregulated in the DHA-treated group while four were downregulated. Notably, six of the seven proteins are enzymes that are important in energy metabolism, with the exception of one molecular chaperone known as HSP60. FH is an important tricarboxylic acid cycle enzyme that catalyzes the hydration of fumarate to form malate and ECAH, located in the matrix of mitochondria, and is a key enzyme that catalyzes the second step in the $\beta$-oxidation pathway of fatty acid metabolism. Both of these enzymes were upregulated following DHA treatment. By contrast, HCAD, which catalyzes the third step of $\beta$-oxidation, two subunits of ATP synthase and NAO, which initiates electron transport for the cytochrome $\mathrm{P} 450$ receiving electrons from NADPH, were downregulated in the DHA-treated group. ATP synthase and NAO are involved in the later steps 
Table I. The mitochondrial proteins with altered expression in BEL-7402 cells after DHA treatment.

\begin{tabular}{|c|c|c|c|c|c|}
\hline Spot & Acc. no. & Protein name & Change & Function & Score \\
\hline 1 & 19743875 & Fumarate hydratase & $\uparrow 2.93$ & $\begin{array}{l}\text { Catalyzes the formation of L-malate from } \\
\text { fumarate }\end{array}$ & 98 \\
\hline 2 & 31542947 & $\begin{array}{l}60 \mathrm{kDa} \text { heat shock } \\
\text { protein }\end{array}$ & $\uparrow 5.19$ & $\begin{array}{l}\text { Essential for the folding and assembly of newly } \\
\text { imported proteins in the mitochondria }\end{array}$ & 138 \\
\hline 3 & 194097323 & Enoyl-CoA hydratase & $\uparrow 1.83$ & $\begin{array}{l}\text { Catalyzes the hydration of 2-trans-enoyl-CoA } \\
\text { intermediates to L-3-hydroxyacyl-CoAs }\end{array}$ & 169 \\
\hline 4 & 83715985 & $\begin{array}{l}\text { 3-hydroxyacyl-CoA } \\
\text { dehydrogenase type-2 } \\
\text { isoform } 2\end{array}$ & $\downarrow 1.82$ & $\begin{array}{l}\text { Catalyzes the oxidation of a wide variety of } \\
\text { fatty acids, alcohols and steroids }\end{array}$ & 131 \\
\hline 5 & 13111901 & ATP5A1 protein & $\downarrow 2.34$ & Catalyzes ATP synthesis & 55 \\
\hline 6 & 51479152 & $\begin{array}{l}\text { ATP synthase subunit } d \text {, } \\
\text { mitochondrial isoform } b\end{array}$ & $\downarrow 3.79$ & Catalyzes ATP synthesis & 142 \\
\hline 7 & 111118981 & $\begin{array}{l}\text { NADPH: adrenodoxin } \\
\text { oxidoreductase }\end{array}$ & $\downarrow 3.89$ & $\begin{array}{l}\text { Initiates electron transport for cytochromes } \\
\text { P450 receiving electrons from NADPH }\end{array}$ & 66 \\
\hline
\end{tabular}

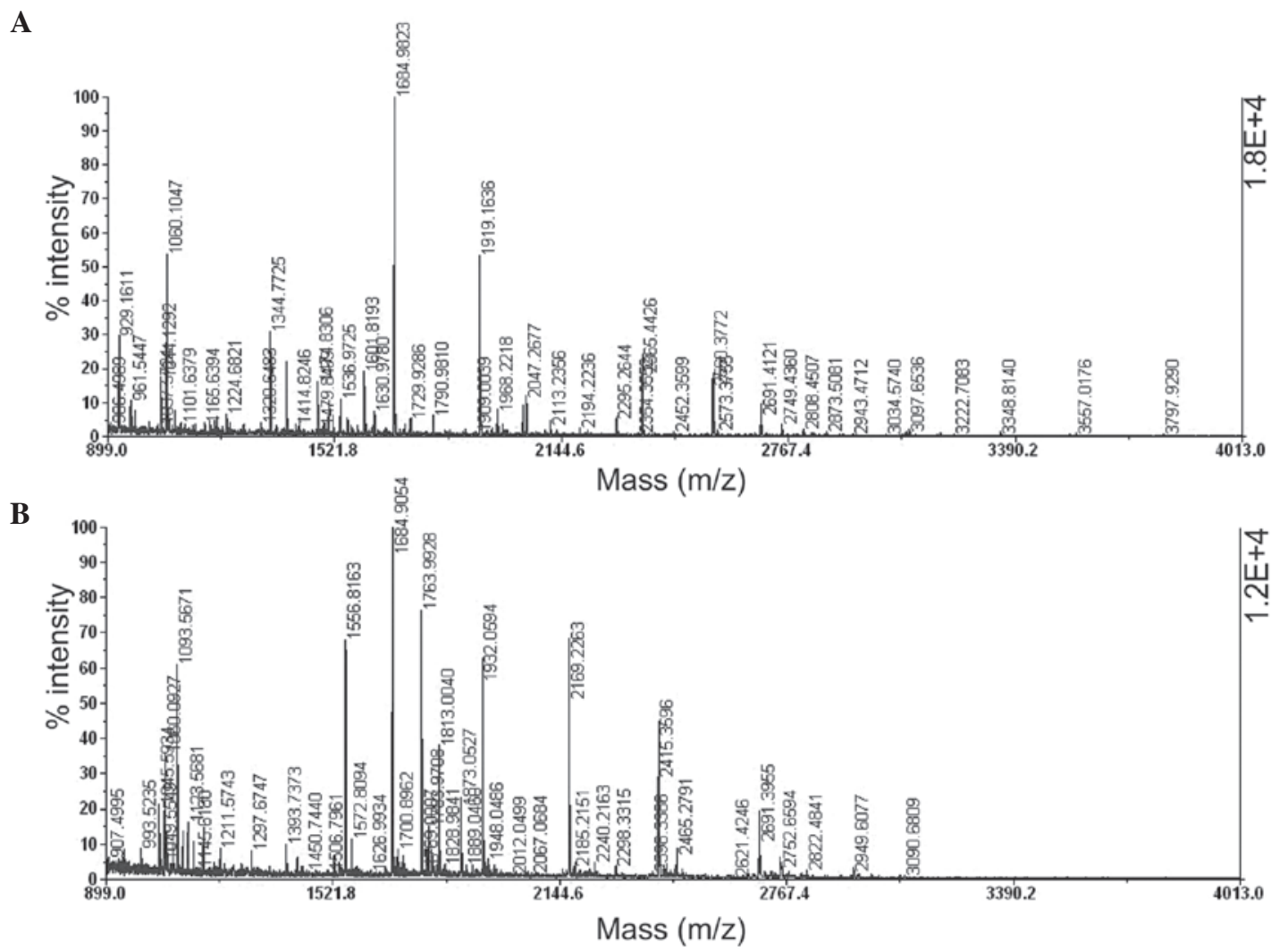

Figure 4. MALDI-TOF-MS peptide maps of in-gel tryptic digest. (A) HSP60 (spot 2) and (B) ATP synthase subunit d (spot 6). MALDI-TOF MS, matrixassisted laser desorption/ionization time-of-flight mass spectrometry.

of ATP production compared with that of FH and ECAH, indicating that the upregulation of $\mathrm{FH}$ and ECAH may be a stimulated response following DHA treatment, while the downregulation of the enzymes of the final ATP production may actually contribute to the anti-cancer potential of DHA. Our hypothesis is further supported by the fact that ARTs, such as artemisinin, artesunate and DHA showed a decrease in intracellular ATP concentration in K562/adr and GLC4/adr cancer cells (19). Another concern is that the ATP synthase is slightly upregulated in HCT116/R cells which are resistant to DHA-induced cell proliferative inhibition (20). All of these findings suggest that energy metabolism is crucial for realizing the anti-cancer effects of DHA. Besides, the biological roles in the signal transduction of these proteins should not be ignored. For example, defects in FH result in hypoxia-inducible transcription factor (HIF) expression, which leads to cancer cell resistance to apoptosis (21). Therefore, the upregulation of $\mathrm{FH}$ following DHA treatment may inhibit the expression of HIF, 
and thus partially contribute to DHA-induced proliferative inhibition in BEL-7402 cells. However, these proteins require further validation with other methods in future studies.

HSP60, a mitochondrial protein that is important for folding key proteins after importing into the mitochondria, was significantly increased in DHA-treated BEL-7402 cells. Similarly, the level of HSP60 was notably increased following exposure of human cancer cells to cisplatin, a chemotherapy drug that causes cross-linking of DNA $(22,23)$. This phenomenon was also demonstrated in human lung non-small carcinoma H460 cells following aloe-emodin treatment (24). However, not all the results are the same. For example, doxorubicin, a topoisomerase II inhibitor, does not cause marked alteration of HSP60 levels in human breast cancer MCF-7 cells (25). Moreover, HSP60 is overexpressed in a number of tumors and plays a pro-survival role in certain cases, suggesting that it is a target for cancer therapy (26). The function of HSP60 in cancer therapy is complicated and the exact role of the upregulation of HSP60 in DHA-treated BEL-7402 cells should be investigated.

It should be mentioned that glucose-regulated protein 78 (GRP78), a protein closely correlated with endoplasmic reticulum stress, was also upregulated, which is consistent with the result obtained from the human colorectal carcinoma HCT116 cells (13). This phenomenon is also observed in human breast cancer MCF-7 cells (data not shown), indicating that DHA-induced endoplasmic reticulum stress may be a universal mechanism in DHA-induced cancer cell proliferative inhibition. However, GRP78 does not transfer into the mitochondria. Several other proteins, such as $70 \mathrm{kDa}$ heat shock protein, protein disulfide isomerase, moesin and maspin (data not shown), were also detected with different expression manners in our experiment, indicating that this method should be further improved for the much more pure mitochondria to be received.

In conclusion, the expression levels of several mitochondrial proteins were changed following DHA treatment and the imbalance of energy metabolism may contribute, at least in part, to the anti-cancer potential of DHA in BEL-7402 cells which may help to shed a new light into DHA-driven events.

\section{Acknowledgements}

We thank Professors Jian Ding and Ying Li for providing the BEL-7402 cell line and DHA, respectively. We also thank Ms. Yu-Fei Chen and Mr. Jun-Ren Zhang for their help with the cell culture and 2-DE assay. This study was supported by the Zhejiang Provincial Education Department (No. Y201016139), National Natural Science Foundation of China (No. 81001450) and Zhejiang Chinese Medicine University (No. 2009ZZ04).

\section{References}

1. White NJ: Qinghaosu (artemisinin): the price of success Science 320: 330-334, 2008.

2. Lu JJ, Meng LH, Cai YJ, et al: Dihydroartemisinin induces apoptosis in HL-60 leukemia cells dependent of iron and p38 mitogen-activated protein kinase activation but independent of reactive oxygen species. Cancer Biol Ther 7: 1017-1023, 2008.

3. Efferth T, Sauerbrey A, Olbrich A, et al: Molecular modes of action of artesunate in tumor cell lines. Mol Pharmacol 64: 382-394, 2003.
4. Efferth T, Olbrich A and Bauer R: mRNA expression profiles for the response of human tumor cell lines to the antimalarial drugs artesunate, arteether, and artemether. Biochem Pharmacol 64: 617-623, 2002.

5. Li LN, Zhang HD, Yuan SJ, Tian ZY, Wang L and Sun ZX: Artesunate attenuates the growth of human colorectal carcinoma and inhibits hyperactive Wnt/beta-catenin pathway. Int J Cancer 121: 1360-1365, 2007.

6. Jiao Y, Ge CM, Meng QH, Cao JP, Tong J and Fan SJ: Dihydroartemisinin is an inhibitor of ovarian cancer cell growth. Acta Pharmacol Sin 28: 1045-1056, 2007.

7. Chen T, Li M, Zhang R and Wang H: Dihydroartemisinin induces apoptosis and sensitizes human ovarian cancer cells to carboplatin therapy. J Cell Mol Med 13: 1358-1370, 2009.

8. Tan W, Lu J, Huang M, et al: Anti-cancer natural products isolated from chinese medicinal herbs. Chin Med 6: 27, 2011.

9. Mercer AE, Maggs JL, Sun XM, et al: Evidence for the involvement of carbon-centered radicals in the induction of apoptotic cell death by artemisinin compounds. J Biol Chem 282: 9372-9382, 2007.

10. Efferth T, Benakis A, Romero MR, et al: Enhancement of cytotoxicity of artemisinins toward cancer cells by ferrous iron. Free Radic Biol Med 37: 998-1009, 2004.

11. Efferth T: Molecular pharmacology and pharmacogenomics of artemisinin and its derivatives in cancer cells. Curr Drug Targets 7: 407-421, 2006.

12. Singh NP and Lai H: Selective toxicity of dihydroartemisinin and holotransferrin toward human breast cancer cells. Life Sci 70: 49-56, 2001.

13. Lu JJ, Chen SM, Zhang XW, Ding J and Meng LH: The anti-cancer activity of dihydroartemisinin is associated with induction of iron-dependent endoplasmic reticulum stress in colorectal carcinoma HCT116 cells. Invest New Drugs 29: 1276-1283, 2011

14. Lu YY, Chen TS, Qu JL, Pan WL, Sun L and Wei XB: Dihydroartemisinin (DHA) induces caspase-3-dependent apoptosis in human lung adenocarcinoma ASTC-a-1 cells. J Biomed Sci 16: 16, 2009.

15. Chen H, Sun B, Pan S, Jiang H and Sun X: Dihydroartemisinin inhibits growth of pancreatic cancer cells in vitro and in vivo. Anticancer Drugs 20: 131-140, 2009.

16. Lu JJ, Meng LH, Shankavaram UT, et al: Dihydroartemisinin accelerates c-MYC oncoprotein degradation and induces apoptosis in c-MYC-overexpressing tumor cells. Biochem Pharmacol 80: 22-30, 2010.

17. Du JH, Zhang HD, Ma ZJ and Ji KM: Artesunate induces oncosis-like cell death in vitro and has antitumor activity against pancreatic cancer xenografts in vivo. Cancer Chemother Pharmacol 65: 895-902, 2010.

18. Li W, Mo W, Shen D, et al: Yeast model uncovers dual roles of mitochondria in action of artemisinin. PLoS Genet 1: e36, 2005.

19. Reungpatthanaphong P and Mankhetkorn S: Modulation of multidrug resistance by artemisinin, artesunate and dihydroartemisinin in K562/adr and GLC4/adr resistant cell lines. Biol Pharm Bull 25: 1555-1561, 2002.

20. Lu JJ, Chen SM, Ding J and Meng LH: Characterization of dihydroartemisinin-resistant colon carcinoma HCT116/R cell line. Mol Cell Biochem 360: 329-337, 2012.

21. King A, Selak MA and Gottlieb E: Succinate dehydrogenase and fumarate hydratase: linking mitochondrial dysfunction and cancer. Oncogene 25: 4675-4682, 2006.

22. Li Z, Zhao X, Yang J and Wei Y: Proteomics profile changes in cisplatin-treated human ovarian cancer cell strain. Sci China C Life Sci 48: 648-657, 2005.

23. Castagna A, Antonioli P, Astner H, et al: A proteomic approach to cisplatin resistance in the cervix squamous cell carcinoma cell line A431. Proteomics 4: 3246-3267, 2004.

24. Lai MY, Hour MJ, Wing-Cheung Leung H, Yang WH and Lee HZ: Chaperones are the target in aloe-emodin-induced human lung nonsmall carcinoma H460 cell apoptosis. Eur J Pharmacol 573: 1-10, 2007.

25. Chen ST, Pan TL, Tsai YC and Huang CM: Proteomics reveals protein profile changes in doxorubicin--treated MCF-7 human breast cancer cells. Cancer Lett 181: 95-107, 2002.

26. Cappello F, Conway de Macario E, Marasa L, Zummo G and Macario AJ: Hsp60 expression, new locations, functions and perspectives for cancer diagnosis and therapy. Cancer Biol Ther 7: 801-809, 2008. 